\title{
Calculation of Solidification of Castings by a Matrix Method*
}

\section{By Itsuo OHNAKA** and Tatsuichi FUKUSAKO**}

\begin{abstract}
Synopsis
A numerical calculation method, "Matrix Method", is proposed for the calculation of solidification of castings for complicated shape, and two examples are shown.

This method has been arrived at by extending Dusinberre's Triangular Network Method and applying the Finite Element Method (FEM). The proposed method has the following advantages over FEM and the conventional Finite Difference Method (FDM).

(1) The physical meaning of the method is clearer than that of FEM.

(2) Various elements, such as triangular element with three or six nodes, triangular ring element, rectangular parallelepiped element and so on, can be more easily employed in this method than in FEM or FDM.

(3) Solidification problems with irregular boundaries can be more easily handled by the present method than by FDM.
\end{abstract}

\section{Introduction}

The temperature distribution, the rate of cooling and the solidification pattern in a casting have considerable influence on the properties of the casting, particularly on shrinkage, segregation, precipitation of inclusion and structure.

For the analysis of solidification problems, analytical, ${ }^{1-4)}$ analogical ${ }^{5-7)}$ and numerical methods ${ }^{8-16)}$ are available. Among these methods, numerical ones have found more general application. Particularly, the Finite Difference Method (FDM) has been widely used. However, FDM is lacking in applicability to the problems with complicated boundaries.

Dusinberre has explained in his book ${ }^{11)}$ a triangular network method for heat conduction problems. Although the method has a possibility to treat irregular boundaries with ease, it has not yet been refined for handy application to those problems. On the other hand, J. G. Henzel, Jr., et al. have reported examples of calculation by applying a method which can handle irregular boundaries with ease. ${ }^{10,12}$ ) In their method, the geometry is divided into cells of any shape or size. It is difficult to discuss their method, because the details have not been reported. Nevertheless, it may be pointed out at least that there should be some limitation, which will be referred to later, to the shape of the cells.

Recently, the Finite Element Method (FEM) has been applied to transient linear heat conduction problems and the solidification problems. ${ }^{17-24}$ In some cases, however, FEM is equivalent in the result to the triangular network method. ${ }^{24}$ ) Therefore, a more convenient calculation method is expected obtainable for solidification problems by extending the triangular network method and applying the algorithm of FEM. The method may be named " matrix method" and is generally available for heat conduction problems, too.

\section{Calculation Method}

\section{Fundamental Conception and Equation}

The basic procedure of the method is as follows:

(1) The geometry of the problem is divided into suitable "elements."

(2) The shape of the element is polygon for oneand two-dimensional problems and polyhedron for three-dimensional problems (See Appendix 1). A "nodal domain" is defined around each " nodal point " which is a vertex of the element. In addition, representative temperature of a nodal domain is assumed to be the "nodal temperature" which is the temperature at the nodal point.

(3) The heat flows from one nodal domain to another proportionally to the temperature difference between the nodal points. In other words, temperature distribution between nodal points is assumed to be approximately linear.

(4) The temperature change which is derivative of temperature with respect to time is determined to satisfy the heat balance equation for each nodal domain. Then, the temperature after a small time increment is obtained from the temperature change.

(5) The liberation of latent heat is treated by the Equivalent Specific Heat Method or Temperature Recovery Method (Iteration Method) mentioned later.

In the procedure above mentioned, it is important that the element and nodal domain are so chosen that the direction of heat flow and the boundary plane of nodal domain intersect each other at right angle. In addition, it is also important to express the heat balance equation in matrix form for each element and superimpose them in an electronic digital computer in order to satisfy the heat balance equation for the whole region concerned.

For example, a triangular element with three nodes as shown in Fig. 1 can be used as the element for oneand two-dimensional problems. In this case, the nodal domains are formed by each side and the perpendiculars from the circumcenter " $\mathrm{O}$ " to the sides. When heat flows through each side, the heat balance equation for nodal domain 1 is given by Eq. (1) (see the top of next page)

* Originally published in The Journal of the Japan Foundrymen's Society, 48 (1976), 139, in Japanese. English version received September 27, 1976

** Faculty of Engineering, Osaka University, Yamada-kami, Suita 565. 
$\rho_{1} c_{1} A_{1} \frac{\partial T_{1}}{\partial t}=\frac{\lambda}{s_{3}} l_{3}\left(T_{2}-T_{1}\right)+\frac{\lambda}{s_{2}} l_{2}\left(T_{3}-T_{1}\right)-\frac{1}{2}\left(s_{2} h_{2}+s_{3} h_{3}\right)\left(T_{1}-T_{a 1}\right)-\frac{\sigma}{2}\left(\varepsilon_{2} s_{2}+\varepsilon_{3} s_{3}\right)\left\{\left(T_{1}+273.15\right)^{4}-\left(T_{a 1}+273.15\right)^{4}\right\}$

$\left[\begin{array}{l}\text { heat ac- } \\ \text { cumlation }\end{array}\right]\left[\begin{array}{l}\text { heat conduc- } \\ \text { tion between }\end{array}\right]\left[\begin{array}{l}\text { heat conduc- } \\ \text { tion between }\end{array}\right]\left[\begin{array}{l}\text { heat convection } \\ \text { through sides } 2 \text { and } 3\end{array}\right]$ points 1 and 2$][$ points 1 and 3$]$

where the thickness of the element is assumed to be unity. From Eq. (1) and two other heat balance equation for nodal domains 2 and 3, one obtains

$$
\begin{aligned}
{[C]\left[\frac{\partial T}{\partial t}\right]=} & {[K][T]-[H]\left[T-\mathcal{T}_{a}\right] } \\
& -[R]\left[(\mathcal{T}+273.15)^{4}-\left(\mathcal{T}_{a}+273.15\right)^{4}\right] \ldots
\end{aligned}
$$

in which

$$
\begin{aligned}
& {[C]=\left[\begin{array}{lll}
\rho_{1} c_{1} A_{1} & & \\
& \rho_{2} c_{2} A_{2} & \\
& & \rho_{3} c_{3} A_{3}
\end{array}\right]} \\
& {[K]=\left[\begin{array}{ccc}
-\Sigma_{1} & l_{3} / s_{3} & l_{2} / s_{2} \\
l_{3} / s_{3} & -\Sigma_{2} & l_{1} / s_{1} \\
l_{2} / s_{2} & l_{1} / s_{1} & -\Sigma_{3}
\end{array}\right]} \\
& {[H]=\frac{1}{2}\left[\begin{array}{ccc}
h_{2} s_{2}+h_{3} s_{3} & & \\
& h_{1} s_{1}+h_{3} s_{3} & \\
& & h_{1} s_{1}+h_{2} s_{2}
\end{array}\right] \text {. }} \\
& {[R]=\frac{\sigma}{2}\left[\begin{array}{lll}
\varepsilon_{2} s_{2}+\varepsilon_{3} s_{3} & & \\
& \varepsilon_{1} s_{1}+\varepsilon_{3} s_{3} & \\
& & \varepsilon_{1} s_{1}+\varepsilon_{2} s_{2}
\end{array}\right]} \\
& {[T]=\left[\begin{array}{c}
T_{1} \\
T_{2} \\
T_{3}
\end{array}\right],\left[\begin{array}{c}
\partial T \\
\partial t
\end{array}\right]=\left[\begin{array}{l}
\partial T_{1} / \partial t \\
\partial T_{2} / \partial t \\
\partial T_{3} / \partial t
\end{array}\right],} \\
& {\left[T-T_{a}\right]=\left[\begin{array}{l}
T_{1}-T_{a 1} \\
T_{2}-T_{a 2} \\
T_{3}-T_{a 3}
\end{array}\right]}
\end{aligned}
$$

where, $\Sigma_{i}$ is the sum of the components of the $i$-th row. In the same manner as Eq. (2), the heat balance equation can be expressed for other elements (See Appendix 2).

By constructing these matrices for each element and superimposing them at the same nodal point, one can assemble one set of linear simultaneous equation for unknown nodal temperature changes over the whole region (Here one can apply the algorithm of FEM). In Fig. 2 (b), for example, nodal point 1 represents the five nodal domains, $a_{1}$ to $e_{1}$, and five heat balance equations are obtained for these domains. The sum

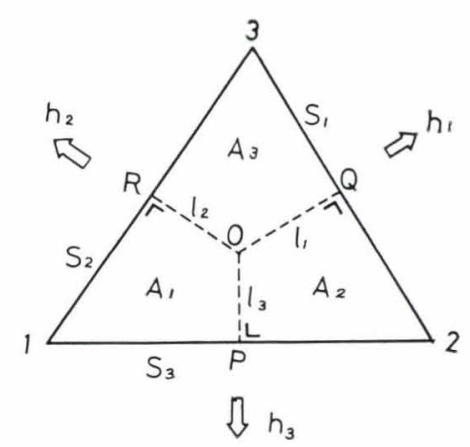

Fig. 1. Triangular element with three nodal points of the left hand sides of the equations gives the total accumulated heat capacity in nodal domain $\left(a_{1}+b_{1}\right.$ $\left.+\cdots+e_{1}\right)$ and the sum of the right hand sides gives the heat conducted from nodal points $2 \sim 6$ to 1 (When an element is not a boundary one, matrices $[H]$ and $[R]$ in Eq. (2) are neglected). Thus, one can see that the heat balance equation is satisfied in the whole region. Note that when the circumcenter of the triangular element does not lie within the element, one can neither define the nodal domain nor satisfy the heat balance equation except for boundary elements (See Appendix 3).

Practically, the left hand side of Eq. (2) is changed to Eqs. (6), (7) and (8) according to Euler's Method (Forward Difference Approximation) as given by Eq. (4) and Modified Euler's Method (Crank-Nicholson's Approximation) as given by Eq. (5):

$$
\begin{aligned}
& \partial T / \partial t=\left(T-T^{B}\right) / \Delta t \ldots \ldots \ldots \ldots \ldots \ldots \ldots \ldots \ldots \ldots(4) \\
& \left(T-T^{B}\right) / \Delta t=\left\{(\partial T / \partial t)^{B}+(\partial T / \partial t)\right\} / 2 \\
& {[C][T]=[C][T]^{B}+\Delta t \cdot[K][T]^{B}-\Delta t[H]\left[T-T_{a}\right]^{B}} \\
& -\Delta t \cdot[R]\left[(T+273.15)^{4}-\left(\mathcal{T}_{a}+273.15\right)^{4}\right]^{B} \\
& \{[C]-\Delta t \cdot[K]+\Delta t \cdot[H]\}[T]=[C][T]^{B} \\
& +\Delta t \cdot[H]\left[T_{a}\right]^{B}-\Delta t \cdot[R]\left[(T+273.15)^{4}\right. \\
& \left.-\left(\mathcal{T}_{a}+273.15\right)^{4}\right]^{B} \text {. } \\
& \left\{\frac{2}{\Delta t}[C]-[K]+[H]\right\}[\mathcal{T}]=\frac{2}{\Delta t}[C][\mathcal{T}]^{B} \\
& +[C]\left[\frac{\partial T}{\partial t}\right]^{B}+[H]\left[T_{a}\right]^{B} \\
& -[R]\left[(T+273.15)^{4}-\left(T_{a}+273.15\right)^{4}\right]^{B} \\
& {[C]\left[\frac{\partial T}{\partial t}\right]^{B}=[K][\mathcal{T}]^{B}-[H]\left[\mathcal{T}-\mathcal{T}_{a}\right]^{B}} \\
& -[R]\left[(T+273.15)^{4}-\left(T_{a}+273.15\right)^{4}\right]^{B}
\end{aligned}
$$

where the variables with superscript $B$ indicate the known values at the time before the time increment $\Delta t$. For the heat radiation term, the known temperature at the time before $\Delta t$ is used in order to linearlize the equation. Although Eq. (6) is inferior to other equations in convergency and accuracy, it may be available in many cases and can save the computing time.

\section{Treatment of Latent Heat}

For the liberation of latent heat during freezing, the Equivalent Specific Heat Method ${ }^{13,14)}$ in which latent heat is transformed into specific heat, and Total Heat Content Method ${ }^{8,16)}$ are widely used. In the present program, however, the Temperature Recovery Method (or Iteration Method) is applied.

In this method, the liquidus temperature of the metal with solid fraction $f_{s}$ is assumed to be

$$
T_{m l}=f_{s} T_{s}+\left(1-f_{s}\right) T_{l}
$$

(Generally $T_{m l}$ is a function of $f_{s}$ ). . . . 
When a nodal temperature drops from $T_{m l}$ to $T_{i}$ $\left(=\mathcal{T}_{m l}-\Delta \mathcal{T}_{i}\right)$ after a time increment $\Delta t$, the temperature is recovered from $T_{i}$ to $T_{m l}$ owing to the liberation of latent heat corresponding to $\Delta T_{i}$. The liberated latent heat, $q_{i}$, and the corresponding solid fraction increment, $\Delta f_{s}$, are calculated by

$$
\begin{aligned}
q_{i} & =\rho c A \Delta T_{i}=\rho L A \Delta f_{s} \\
\Delta f_{s} & =c \Delta T_{i} / L \quad \ldots \ldots \ldots \ldots \ldots \ldots
\end{aligned}
$$

where, $A$ is the area or volume of the nodal domain. The solid fraction is given by

$$
f_{s}=\Sigma \Delta f_{s}
$$

In the solid-liquid region, the procedure mentioned above is repeated either until $f_{s}$ becomes 1.0 in Eq. (12) or until Eq. (13) is satisfied:

$$
\Sigma q_{i}=\rho c A \Sigma \Delta T_{i}=\rho L A \Sigma \Delta f_{s}=\rho L A
$$

In this method, the latent heat may be evaluated more accurately than in the Equivalent Specific Heat Method, and the liquidus temperature change owing to solute enrichment can easily be taken into account.

\section{Material 2}

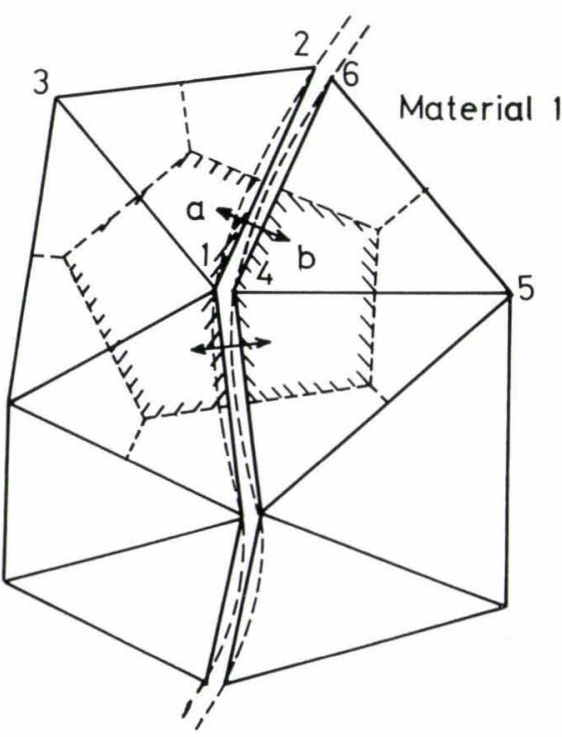

Fig. 2(a). Dividing at the boundary with heat resistance

\section{Material 2}

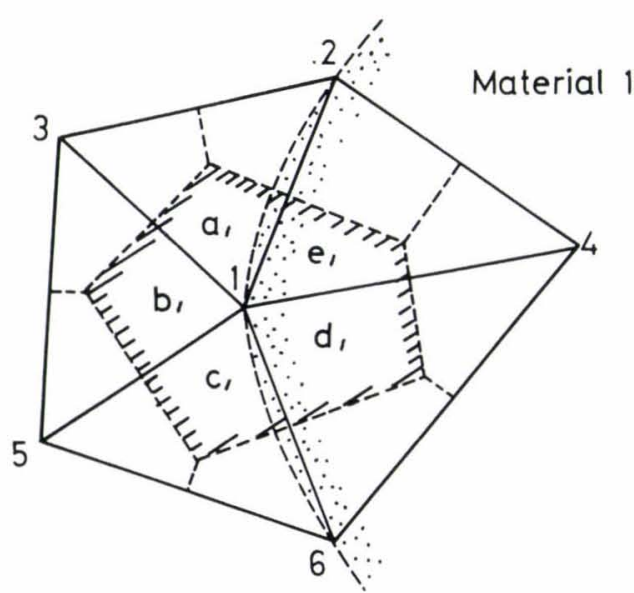

Fig. 2(b). Dividing at the boundary without heat resistance

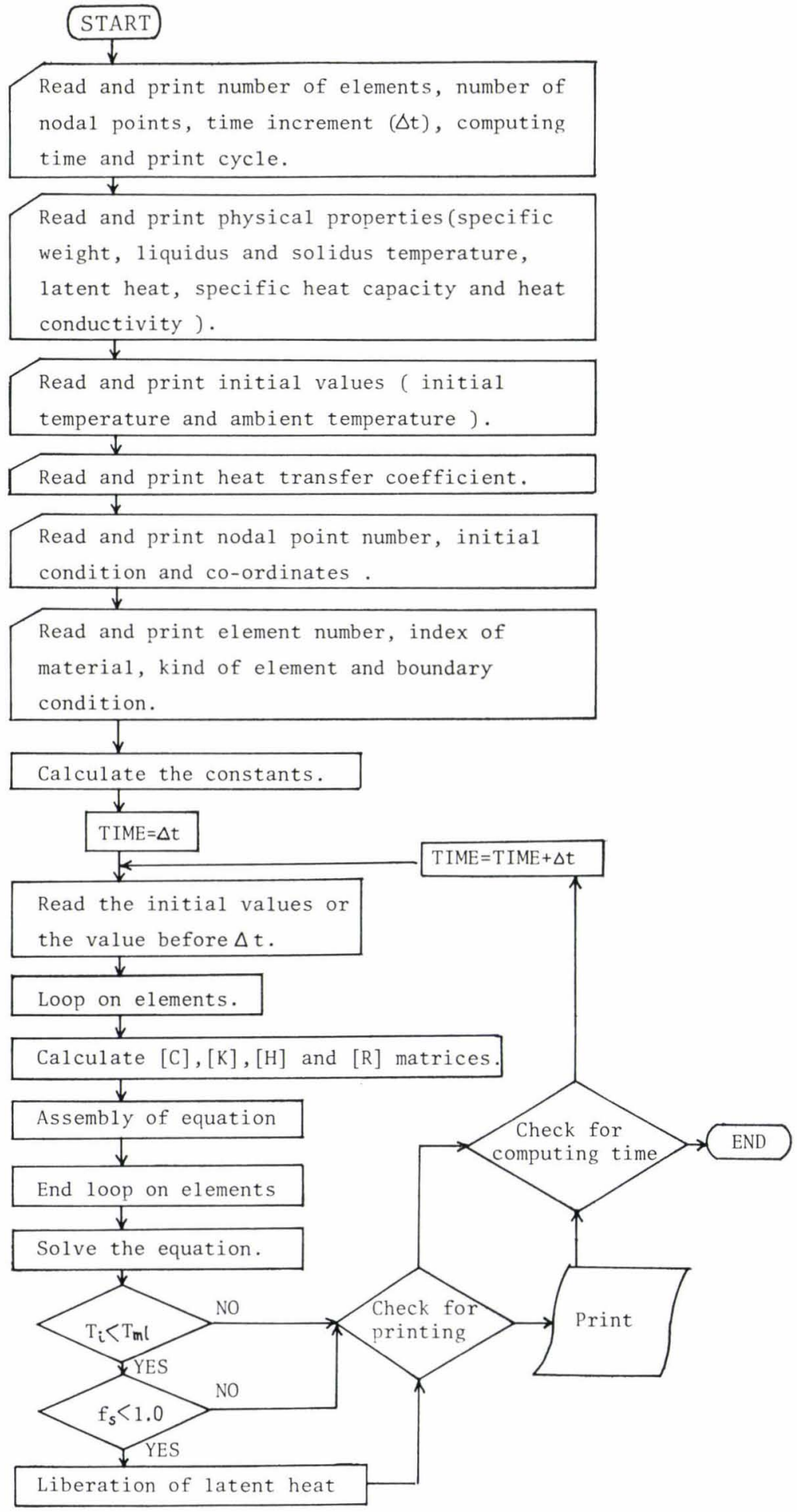

Fig. 3. Flow chart of the program 
However, this method is essentially equivalent to the Equivalent Specific Heat Method and the Total Heat Content Method.

\section{Boundary and Initial Condition}

In the calculation of solidification of castings, the boundary condition at the mold-casting interface and the treatment of the riser and the surface of molten metal are important. However, in this program only the condition at the mold-casting and mold-atmosphere interface are considered as follows:

In case there is a heat resistance between casting and mold, we take one pair of nodal points at the mold-casting interface. The heat transfer occurs between the nodal points as shown in Fig. 2 (a). Here nodal temperatures $T_{4}$ and $T_{6}$ are regarded as the ambient temperatures for nodal temperatures $T_{1}$ and $T_{2}$. The other nodal point of the pair is named " corresponding nodal point at boundary."

When there is no heat resistance, the region near the boundary is divided as shown in Fig. 2 (b). Although in this case more than two kinds of material are mixed in the same nodal domain (for example in nodal domain 1), the authers think this unavoidable for the simplification of dividing.

For the nodal points on the surface of mold or metal, proper ambient temperatures are designated. The heat transfer coefficient can be varied with time or temperature.

As the initial value at each nodal point an arbitrary value can be designated.

\section{Flow Chart of the Program and Preparation of the Input Data}

Three programs have been made for one-, two- and three-dimensional, and axi-symmetrical problems.
The programs are written in Fortran IV. The total number of cards for the program for three-dimensional problems is about 700 and the necessary memory size is $324 \mathrm{kch}$ for 500 nodal points. The flow chart of the programs is shown in Fig. 3. These programs have more than 15 subroutines. The physical properties and heat transfer coefficients can be varied with temperature or time.

As expected from the flow chart, this method requires more complicated and more input data than the conventional finite difference method. In Fig. 4, examples of input data sheets are shown. Especially, the dividing and numbering in three-dimensional analyses require many hours. In the authers' technique, the domain interested is cut into slices. The slices are then divided into appropriate elements and numbered. Since the dividing and numbering in this method are the same as those in FEM, programs for automatic dividing in FEM may be applied, too.

\section{Examples of Calculation}

Figure 5 shows a three-dimensional analyses of steel casting $(0.29 \% \mathrm{C}, 0.62 \% \mathrm{Si})$ in a $\mathrm{CO}_{2}$ mold. In this case, rectangular parallelepiped and triangular prism elements were employed. The heat resistance between mold and casting was ignored and the initial temperature at the interface was supposed to be equal to the liquidus temperature $\left(1510^{\circ} \mathrm{C}\right)$. The pouring temperature was $1650^{\circ} \mathrm{C}$. The physical data used in the calculation are shown in Appendix 4. As seen in Fig. 5 (b), the calculated temperature decreases faster than measured one. This would result from the formation of air gap between mold and casting. The computation was made using NEAC-2200 Model 700 at the Computation Center of Osaka University. The computing time was $190 \mathrm{sec}$ for $1 \mathrm{sec}$ time increment, 175
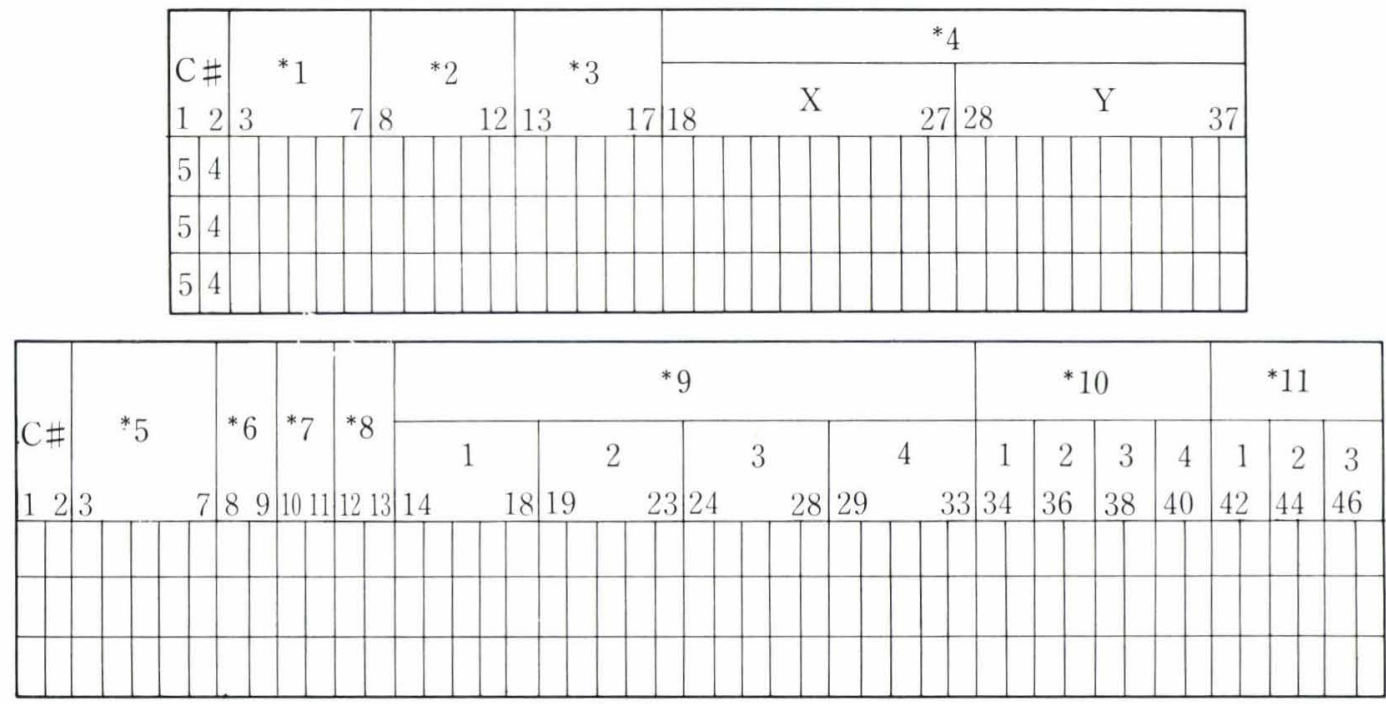

${ }^{*} 1$; Nodal point number

${ }^{*} 2$; Initial value index

*3; Corresponding nodal point number at boundary
${ }^{*} 5$; Element number ${ }^{*} 9$; Nodal point numbers of element

${ }^{*} 6$; Material index ${ }^{*} 10$; Boundary surface number and

${ }^{*} 7$; Shape index

$* 8$; Boundary heat transfer coefficient index

${ }^{*} 11$; Heat radiation surface number

${ }^{*} 4$; Co-ordinates of nodal point

Fig. 4. Examples of imput sheet data (for axi-symmetric solids) 


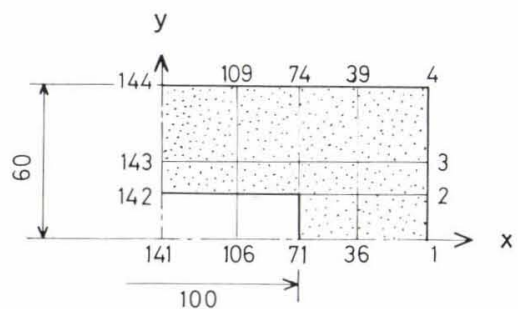

148 Elements

175 Points
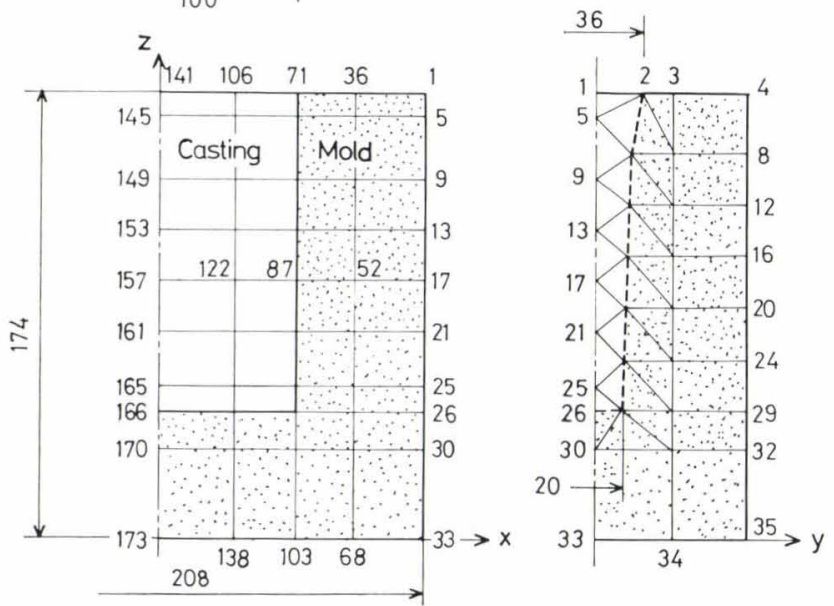

Fig. 5(a). Three-dimensional analysis of plate casting by $\mathrm{CO}_{2}$ mold (Steel casting, Dimensions in $\mathrm{mm}$ )

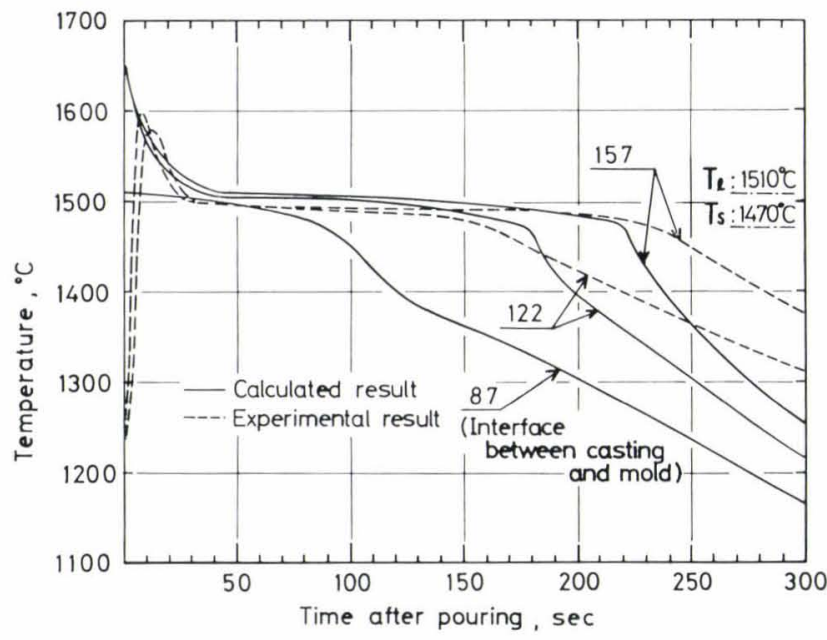

Fig. 5(b). Comparison of calculated result with measured values (Numbers indicate nodal points)

nodal points, 148 elements and $300 \mathrm{sec}$ analysis time.

Figure 6 shows an analysis of steel ingot $(0.2 \% \mathrm{C}$, $0.62 \% \mathrm{Mn}, 0.03 \% \mathrm{Si}$ ) in a cast iron mold. Triangular ring element and rectangular ring element were employed in the analysis. It was assumed that heat exchange between mold and ingot occurs due to radiation across and convection in the air gap. The heat transfer coefficient was assumed to be $72 \mathrm{kcal} /\left(\mathrm{m}^{2} \cdot \mathrm{h}\right.$ -deg). The calculated result agrees well with the experimental one. It would be said that radiation influences considerably on the solidification of large ingots. The computing time was about $30 \mathrm{~min}$ for 3 sec time increment, 212 nodal points, 206 elements and $4 \mathrm{hr}$ analysis time.

In the above mentioned examples, Eq. (6) was ap-

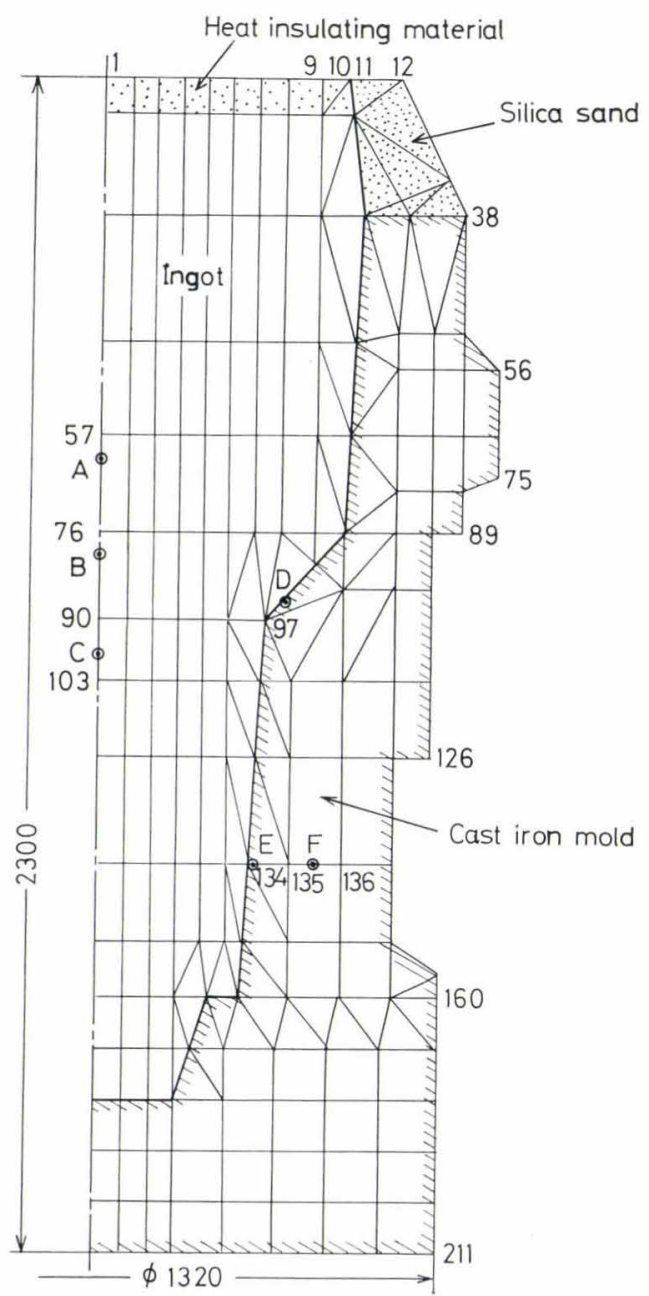

Fig. 6(a). Analysis of $6.5 \mathrm{t}$ steel ingot (Numbers indicate nodal points and the alphabet measuring point, Dimensions in $\mathrm{mm}$ )

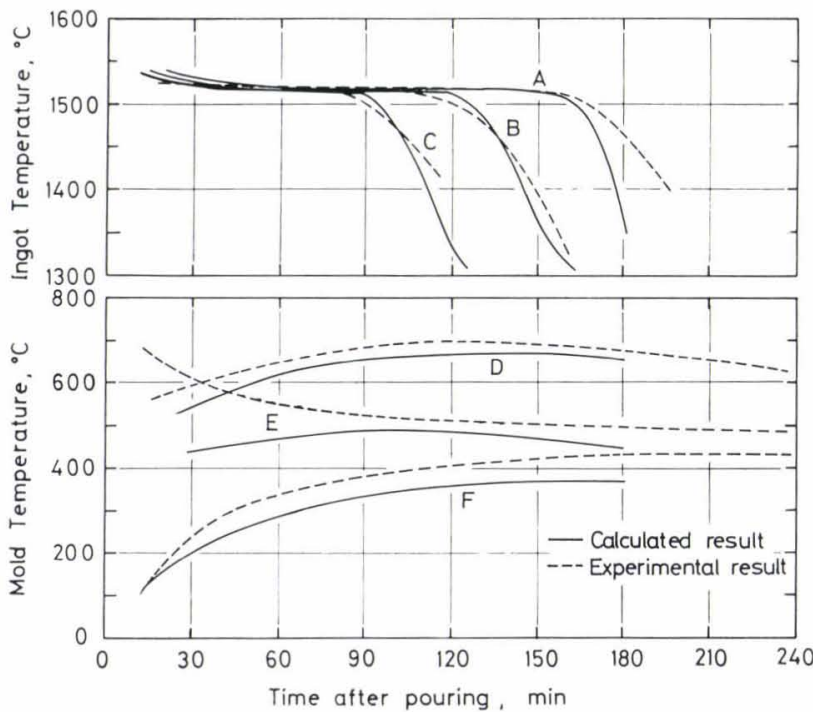

Fig. 6(b). Comparison of calculated result with measured values.

plied and the initial temperature distribution within metal and mold was ignored. The calculation was started at the time when the melt filled the mold. 


\section{Conclusion}

As seen from the examples, the proposed method can be usefully applied to the analysis of solidification problems with irregular boundaries. It may also become a useful one for thermal stress analysis. The accuracy of the method would be the same as that of the conventional FDM because the principle is the same. The computing time of the proposed method is almost the same as that of FEM ${ }^{24)}$ and about ten times as long as that of FDM. The greatest disadvantage of the proposed method as compared with FDM is that it requires many input data particularly in three-dimensional analyses. Rationalizing the method for preparing the input data, therefore, is of almost importance. It is also hoped to study the treatment of temperature drop during pouring, heat resistance between mold and casting, thermal properties of sand mold, and accuracy of calculation due to dividing.

\section{Nomenclature}

$c:$ specific heat $\left(\mathrm{kcal} \cdot \mathrm{kg}^{-1} \cdot \mathrm{deg}^{-1}\right)$

$f_{s}$ : solid fraction

$h$ : heat transfer coefficient $\left(\mathrm{kcal} \cdot \mathrm{m}^{-2} \cdot \mathrm{sec}^{-1}\right.$ $\cdot \operatorname{deg}^{-1}$ )

$l:$ a line through which heat conduction occurs (m)

$s:$ side length $(\mathrm{m})$

$q$ : liberated latent heat (kcal)

$t:$ time (sec)

$\Delta t$ : time increment (sec)

$A$ : area $\left(\mathrm{m}^{2}\right)$

$[C]$ : heat capacity matrix

$F$ : a plane through which heat conduction occurs $\left(\mathrm{m}^{2}\right)$

$[H]$ : heat transfer matrix

$[K]$ : heat conduction matrix

$L:$ latent heat $\left(\mathrm{kcal} \cdot \mathrm{kg}^{-1}\right)$

$[R]$ : heat radiation matrix

T: temperature $\left({ }^{\circ} \mathrm{C}\right)$

$T_{l}, T_{s}:$ liquidus and solidus temperature, respectively

$T_{m \ell}$ : liquidus temperature in the solid-liquid region

$V:$ volume $\left(\mathrm{m}^{3}\right)$

$\varepsilon$ : heat radiation coefficient

$\lambda$ : heat conductivity $\left(\mathrm{kcal} \cdot \mathrm{m}^{-1} \cdot \mathrm{sec}^{-2} \cdot \mathrm{deg}^{-1}\right)$

$\rho: \quad$ specific gravity $\left(\mathrm{kg} \cdot \mathrm{m}^{-3}\right)$

$\sigma$ : Stefan-Boltzmann constant $\left(\mathrm{kcal} \cdot \mathrm{m}^{-2} \cdot \mathrm{sec}^{-1}\right.$ $\left.\cdot \mathrm{K}^{-4}\right)$

Subscripts

$1,2, \ldots$ : number of nodal points

a : ambient

Superscript

$B:$ a value before the time increment $\Delta t$

\section{Acknowledgements}

The authers wish to acknowledge helpful discussions with Prof. Dr. Y. Yamamoto, Prof. Dr. K. Chijiiwa of Tokyo University and Mr. H. Tanizawa of Kubota Ltd. They also wish to express thanks to the engineers of Hitachi Shipbuilding \& Engineering
Co., Ltd. for the temperature measurements of the steel ingot.

\section{REFERENCES}

1) H. S. Carslaw and J. C. Jaeger: Conduction of Heat in Solids, Oxford, (1959), 282.

2) A. W. Hills: Trans. Met. Soc. AIME, 245 (1969), 1471.

3) H. Jones: J. Inst. Metals, 97 (1969), 38.

4) J. C. Muehlbauer, J. D. Hatcher, D. W. Lyons and J. E. Sunderland: J. Heat Transfer, Trans. ASME-C, 95 (1973), 324.

5) V. Paschkis: Trans. Amer. Found. Soc., 59 (1951), 7.

6) V. Paschkis: Trans. Amer. Found. Soc., 61 (1953), 142.

7) K. Chijiiwa, M. Ito, M. Kato and M. Iwasaki: Giesserei, 58 (1971), 570 .

8) R. J. Sarjant and M. R. Slack: JISI, 177 (1954), 428.

9) W. D. Murray and F. Landis: J. Heat Transfer, Trans. ASME-C, 81 (1959), 106.

10) D.J. Campbell and D. B. Vallemweider: Proc. Eastern Joint Computer Conference, 16 (1959), 143.

11) G. M. Dusinberre: Heat Transfer Calculations by Finite Differences, International Textbook Co., (1961), 89.

12) J. G. Henzel, Jr. and J. Keverian: Trans. Amer. Found Soc., 74 (1966), 661.

13) Eugene A. Mizikar: Trans. Met. Soc. AIME, 239 (1967), 1747.

14) K. Katayama and M. Hattori: Trans. Japan Soc. Mech. Eng., 40 (1974), 333.

15) J. Alan Adams and David F. Rogers: Computer-Aided Heat Transfer Analysis, McGraw-Hill, (1973), 182.

16) V. de L. Davies, S. Stokke and O. Westby: The British Foundryman, 66 (1973), 305.

17) O. C. Zienkiewicz: The Finite Element Method in Engineering Science, McGraw-Hill, (1971). 322.

18) T. Fujino: J.Japan Soc. Mech. Engrs., 94 (1971), 673.

19) T. Iwaki: Proc. the 7th Seminar on Matrix Methods of Structural Analysis and Design, Soc. Steel Construction of Japan, (1973), 649.

20) M. Shoji, T. Sasaki, K. Imai and T. Narita: Proc. the 7 th Seminar on Matrix Methods of Structural Analysis and Design, Soc. Steel Construction of Japan, (1973), 665.

21) O. C. Zienkiewicz: Lectures on Finite Element Methods in Continuum Mechanics, ed. by J. T. Oden and E. R. A. Oliveira, UAH Press, Alabama, (1973), 379.

22) O. C. Zienkiewicz and C. Taylor: Lectures on Finite Element Methods in Continuum Mechanics, ed. by J. T. Oden and E. R. A. Oliveira, UAH Press, Alabama, (1973), 439.

23) J. I. Soliman and E. A. Fakhroo: J. Mech. Eng. Science, 14 (1972), 19.

24) I. Ohnaka and T. Fukusako: Tech. Rept. Osaka University, 24 (1974), 461.

\section{Appendix}

\section{Elements Used in the Matrix Method}

Figure A-1 shows the available elements for oneand two-dimensional analyses, and Fig. A-2 shows typical elements for three-dimensional analyses. In these figures, nodal points and nodal domains are illustrated, too. The nodal domains are always formed by surface planes of the element and its bisectional planes. Ring elements and prisms, the cross sections of which are illustrated in Fig. A-1, may be used for axi-symmetrical and three-dimensional problems, respectively. 


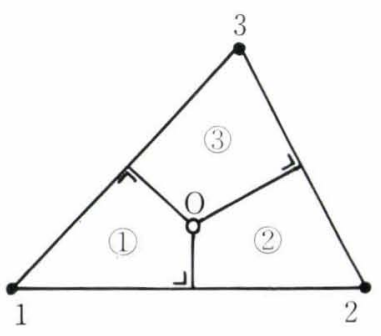

(a)

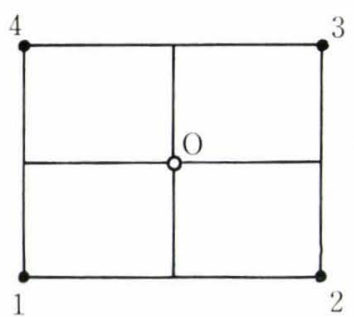

(e)

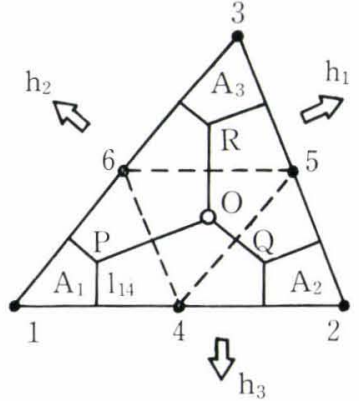

(b)

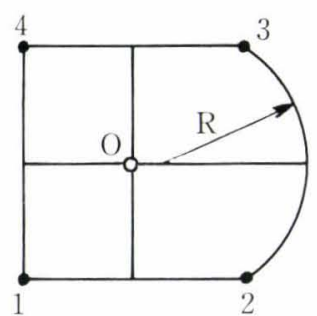

(f)

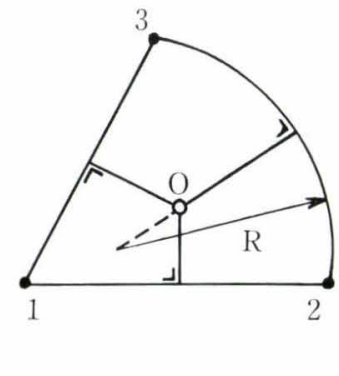

(c)

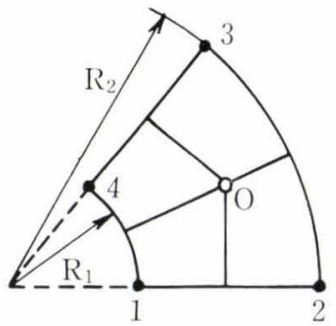

(g)

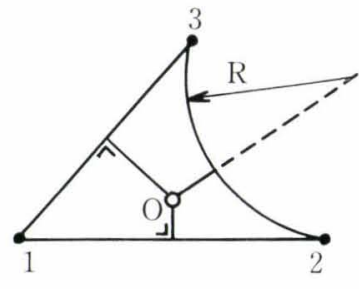

(d)

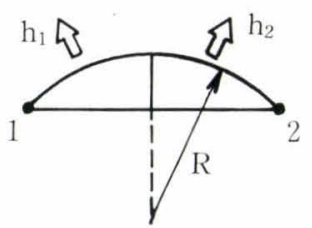

(h)

Fig. A-1. Various elements used in one- and two-dimensional problems (Numbers indicate nodal points)

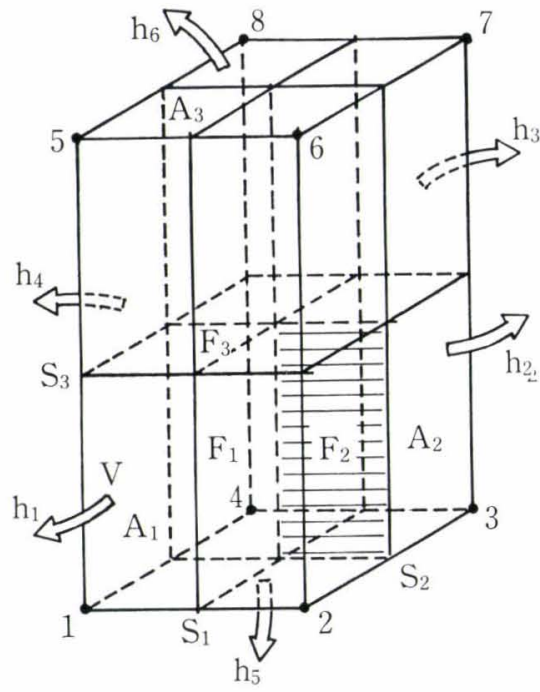

(a) Rectangular parallelepiped element

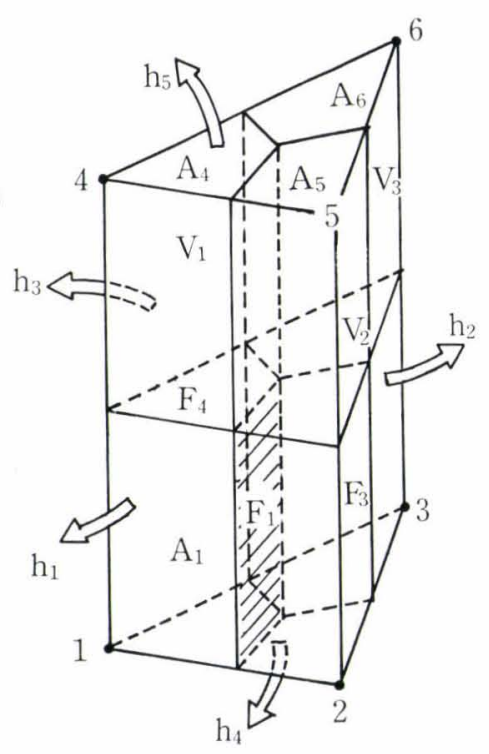

(b) Triangular prism element

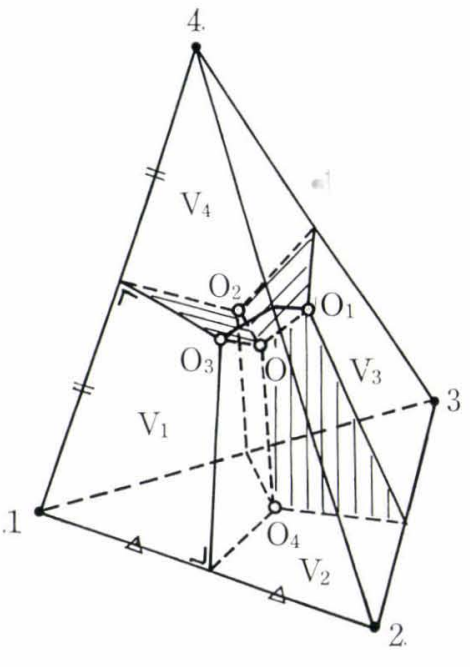

(c) Tetrahedron element

Fig. A-2. Typical elements used in three-dimensional problems (Numbers indicate nodal points)

In these elements, the tetrahedron element $((c)$ in Fig. A-2) may not be usually used, because the element should have its circumcenter within itself but it is hard to assure it. The segment element $((h)$ in Fig. A-1) should also be used only at the boundary between mold and atmosphere because of its inaccuracy. (At the boundary, heat transfer is generally more important than heat conduction.)

\section{Heat Balance "Matrices" for Some Elements}

1. Triangular Element with Six Nodal Points (Fig. A-1 (b))

The nodal points, 4,5 and 6 , lie at the bisection points of the sides. The points, $O, P, Q$ and $R$, are circumcenters of triangles formed by the broken lines and the sides. By employing this element, we can reduce the imput data by about one-half. The matrices in Eq. (2) are shown in Eq. (A-1) (The $[R]$ matrix is omitted because of insufficient space):

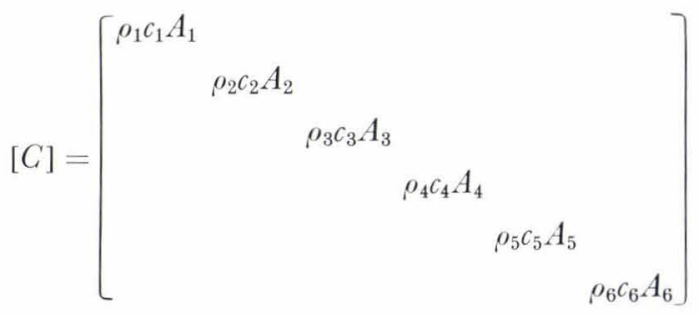




$$
[K]=2 \lambda\left[\begin{array}{cccccc}
-\Sigma_{1} & 0 & 0 & l_{14} / s_{12} & 0 & l_{16} / s_{13} \\
& -\Sigma_{2} & 0 & l_{24} / s_{12} & l_{25} / s_{23} & 0 \\
& & -\Sigma_{3} & 0 & l_{35} / s_{23} & l_{36} / s_{13} \\
& & & -\Sigma_{4} & l_{45} / s_{45} & l_{46} / s_{46} \\
& \text { Sym. } & & & -\Sigma_{5} l_{56} / s_{56} \\
& & & & & -\Sigma_{6}
\end{array}\right]
$$

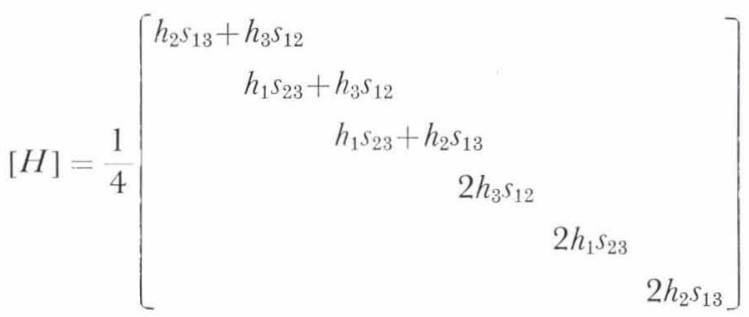

(A-1-c)

where, $\Sigma_{i}$ denotes the sum of the elements in the $i$-th row, $l_{i j}$ denotes the length through which heat conduction between points $i$ and $j$ occurs and $s_{i j}$ denotes the length between points $i$ and $j$.

\section{Rectangular Parallelepiped Element with 8 Nodal Points (Fig. A-2(a))}

In this case, the nodal domains are formed by bisectional planes of each surface plane and the surface planes of the element. The matrices, $[C],[K]$ and $[H]$, are as follows (Eqs. A-2-a $\sim$ c):
3. Triangular Ring Element with 3 Nodal Points (Fig. A-1)

The $[C],[K],[H]$ matrices are as follows:

$$
\begin{aligned}
& {[C]=2 \pi\left[\begin{array}{lll}
\rho_{1} c_{1} r_{1}^{*} A_{1} & & \\
& \rho_{2} c_{2} r_{2}^{*} A_{2} & \\
& & \rho_{3} c_{3} r_{3}^{*} A_{3}
\end{array}\right] \ldots \ldots(\mathrm{A}-3-\mathrm{a})} \\
& {[K]=2 \pi \lambda\left[\begin{array}{ccc}
-\Sigma_{1} & l_{3}^{*} l_{3} / s_{3} & l_{2}^{*} l_{2} / s_{2} \\
-\Sigma_{2} & l_{1}^{*} l_{1} / s_{1} \\
\text { Sym. } & -\Sigma_{3}
\end{array}\right]} \\
& {[H]=\pi\left[\begin{array}{c}
s_{2}^{*} s_{2} h_{2}+s_{3}^{*} s_{3} h_{3} \\
s_{1}^{*} s_{1} h_{1}+s_{3}^{*} s_{3} h_{3} \\
s_{1}^{*} s_{1} h_{1}+s_{2}^{*} s_{2} h_{2}
\end{array}\right]}
\end{aligned}
$$

in which $r_{i}^{*}$ denotes the co-ordinate of the center of gravity of each nodal domain in the radial direction, $l_{i}^{*}$ denotes the co-ordinate of the bisectional point of $l_{i}$ in the radial direction and $s_{i}^{*}$ denotes the half of $s_{i}$.

\section{Some Exceptions with Respect to Element}

Elements which do not have their circumcenter within themselves (see Fig. A-3) can be used only when side $\overline{23}$ of Fig. A-3 (a) or (b) faces to atmosphere. Otherwise, correct nodal domain cannot be formed. Taking for example Fig. A-3 (c), we cannot consider heat conduction through $\mathrm{PQ}$. This causes an error. When the element faces to atmosphere, we

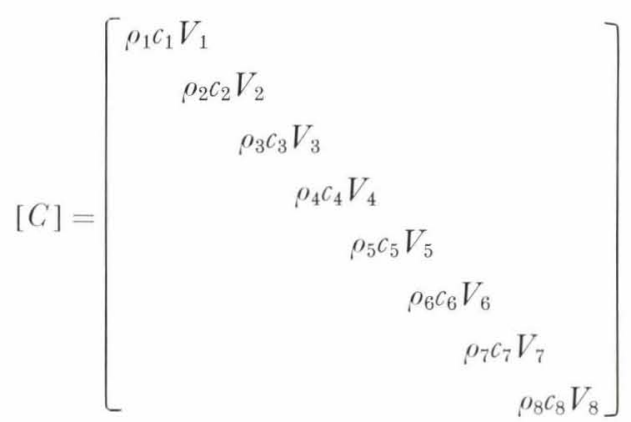

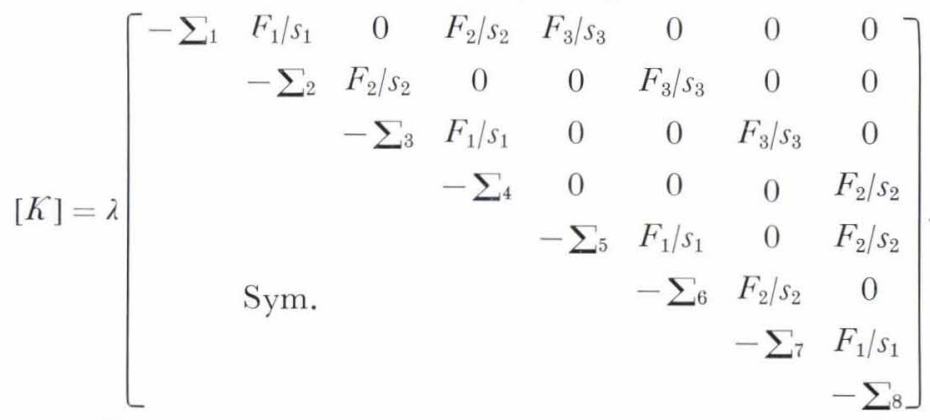

$$
[H]=\left[\begin{array}{r}
h_{1} A_{1}+h_{4} A_{2}+h_{5} A_{3} \\
h_{1} A_{1}+h_{2} A_{2}+h_{5} A_{3} \\
h_{2} A_{2}+h_{3} A_{1}+h_{5} A_{3} \\
h_{3} A_{1}+h_{4} A_{2}+h_{5} A_{3} \\
h_{1} A_{1}+h_{4} A_{2}+h_{6} A_{3} \\
h_{1} A_{1}+h_{2} A_{2}+h_{6} A_{3} \\
h_{2} A_{2}+h_{3} A_{1}+h_{6} A_{3} \\
h_{3} A_{1}+h_{4} A_{2}+h_{6} A_{3}
\end{array}\right]
$$


can consider heat transfer through $\overline{23}$ and neglect heat conduction between nodal points 2 and 3 . This causes no inconsistency.

Fig. A-3. Exceptional elements (O denotes circumcenter)

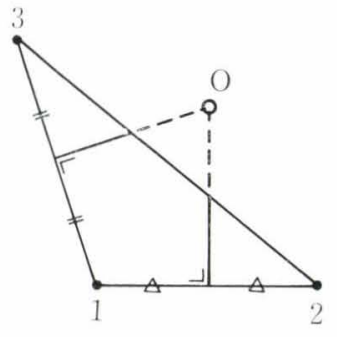

(a)

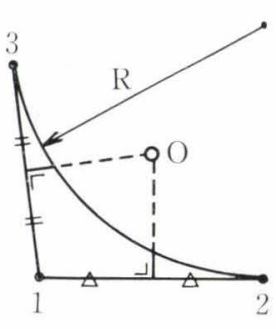

(b)

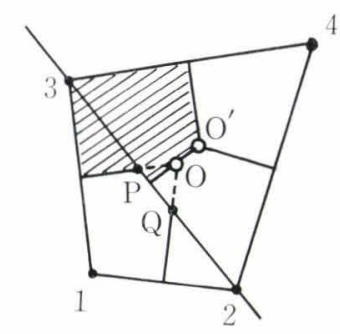

(c)

4 Physical Data for Materials Employed

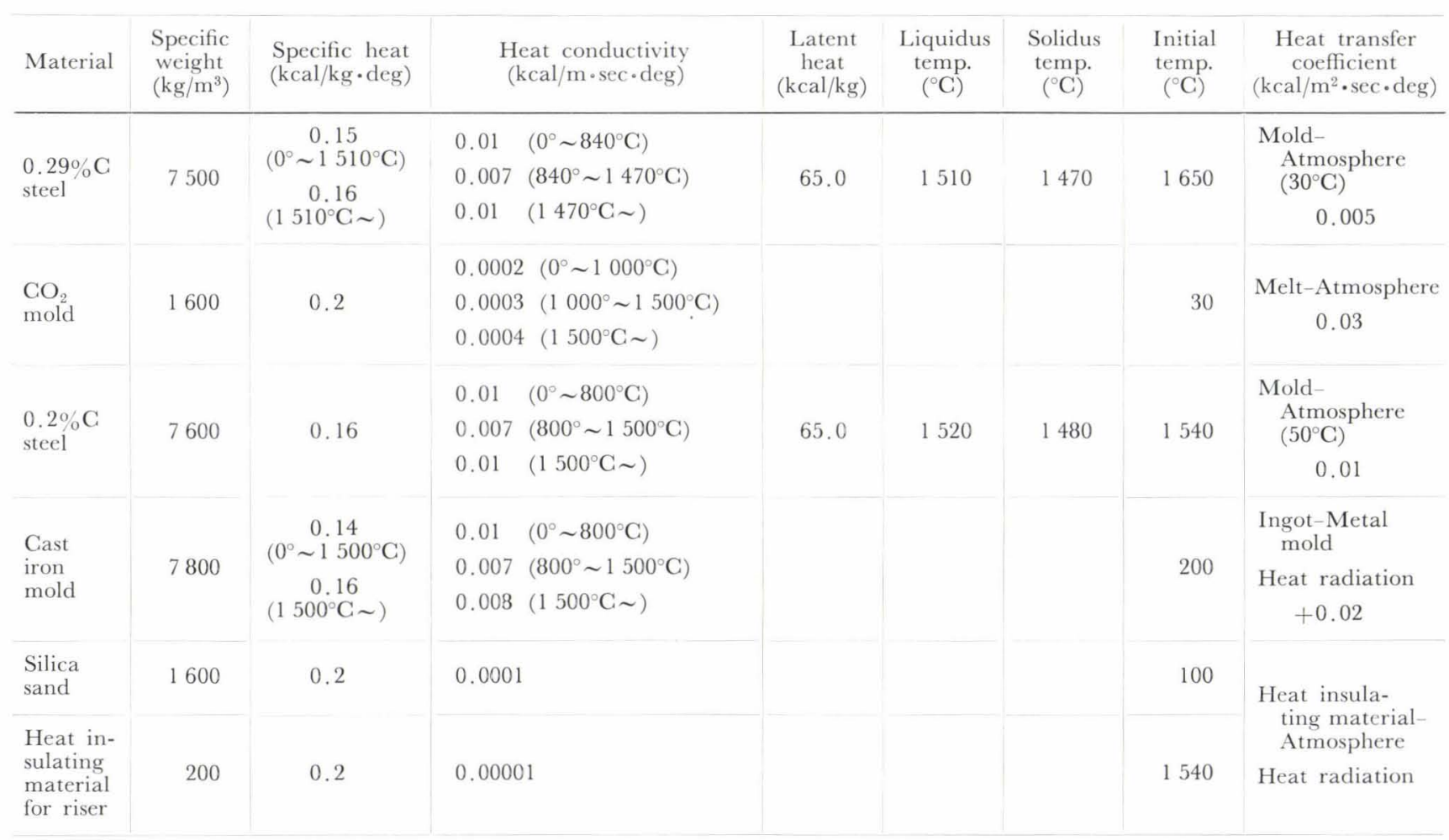

